Systematic Review

\title{
Efficacy of Pain Relief in Different Postherpetic Neuralgia Therapies: A Network Meta-Analysis
}

Dehua Song, MD'1 Aijie He, MD², Renhua Xu, $\mathrm{PhD}^{3}$, Xiaolin Xiu, BA ${ }^{4}$, and Yulian Wei, BA

From: ${ }^{1}$ Department of Radiotherapy, The Affiliated Yantai Yuhuangding Hospital of Qingdao University, Shandong, China; ${ }^{2}$ Department of Neurosurgery, The

Affiliated Yantai Yuhuangding Hospital of Qingdao University, Shandong, China;

${ }^{3}$ School of Nursing, Binzhou Medical University, Shandong, China; ${ }^{4}$ Department of Reproductive Medicine, The Affiliated Yantai Yuhuangding Hospital of Qingdao University, Shandong, China; ${ }^{5}$ Department of Joint Orthopaedic Surgery, The Center Hospital of Zibo, Shandong, China

Address Correspondence: Yulian Wei, BA Department of Joint Orthopaedic Surgery, The Center Hospital of Zibo No.54 Gongqingtuan Rd Zhangdian District, Zibo 255036, Shandong, China E-mail: zc_yangxiufei@163.com

Disclaimer: Dehua Song and Aijie He are first co-authors.

Conflict of interest: Each author certifies that he or she, or a member of his or her immediate family, has no commercial association (i.e., consultancies, stock ownership, equity interest, patent/licensing arrangements, etc.) that might pose a conflict of interest in connection with the submitted manuscript.

Manuscript received: $12-20-2016$ Revised manuscript received: 05-18-2017

Accepted for publication: 05-30-2017

Free full manuscript: www.painphysicianjournal.com
Background: Postherpetic neuralgia (PHN) is a nerve pain disease usually controlled by different therapies, i.e., topical therapies, antiepileptics, analgesics, antipsychotics, antidepressants, anti-dementia drugs, antivirals, amitriptyline, fluphenazine, and magnesium sulfate. It is believed that different therapies may lead to different levels of pain relief.

Objectives: We proposed this study to compare the efficacy of PHN treatments.

Study Design: We conducted a systematic review of the current literature. All relevant studies were retrieved from online databases. The standardized mean difference (SMD) was used for pain relief measurement in different PHN therapies.

Setting: A conventional meta-analysis and a network meta-analysis (NMA) were carried out together with the surface under the cumulative ranking curve (SUCRA) for each therapy calculated regarding their efficacy.

Results: A pairwise meta-analysis suggested that 4 treatment classes, including topical therapies, antiepileptics, analgesics, and antidepressants, exhibited better pain relief results than placebo. Likewise, a NMA suggested that patients with 4 treatment classes exhibited significant improvements in pain scores compared to those with placebo.

Limitations: There is a lack of direct head-to-head comparisons of some treatments, especially for antivirals, anti-dementia drugs, and magnesium sulfate. Secondly, the specific agents belonging to the same class of therapies might exhibit different effects (gabapentin and carisbamate) with different mechanisms (opioids and ketamine) on reducing pain, and some agents were hard to find in literatures and were not involved in our study, which may influence our results.

Conclusions: Analgesics were preferable to other treatments with respect to pain relief for PHN, while antivirals appeared to be less effective than other therapies.

Key words: Postherpetic neuralgia, topical agents, antiepileptics, analgesics, antipsychotics, antidepressants

Pain Physician 2018; 21:19-32
$D$ ostherpetic neuralgia (PHN) is a common type of neuropathic pain syndrome, characterized as pain persisting for at least 120 days at the site of acute herpes zoster subsequent to rash onset (1). It has been reported that approximately $10-20 \%$ of patients with herpes zoster will develop PHN, and there is a positive correlation between the severity/ prevalence of PHN and the age of patients $(2,3)$. Pain associated with PHN may result from direct neuronal damage to the peripheral and central nervous systems. PHN is usually accompanied with dysesthesia, paresthesia, allodynia, and hyperalgesia (4-6). These syndromes, which usually last for many years, can severely disturb the sleep, alter the mood, and affect the quality of life of patients. 
There are 2 groups of therapies usually prescribed for PHN patients: topical therapies (lidocaine and capsaicin) and systemic therapies (7). Topical agents, such as lidocaine, are formulated to provide patients with local pain relief while minimizing systemic absorption (8). In addition, NGX-4010, a high-concentration capsaicin $(8 \%)$, is reported to relieve pain for up to 12 weeks in PHN patients (9). Systemic therapies include antiepileptics, analgesics, antipsychotics, antidepressants, anti-dementia drugs, and magnesium sulfate (7). In addition, the varicella zoster virus vaccine was able to reduce the incidence of herpes zoster as well as PHN, indicating that antivirals may be effective for PHN patients (10). A combination therapy of amitriptyline and fluphenazine (A\&F) also showed its efficacy for PHN patients (11). However, relative efficacy of these therapies remains unclear (12). As a result, evaluation and comparison for these potential therapies with respect to efficacy is essential in clinical practice.

A variety of previous studies have assessed the efficacy of PHN treatments. However, the majority of these studies were trials or reviews, which only provided direct comparisons $(13,14)$. Apart from that, the power of some studies was limited by a small sample size, which may provide insignificant conclusions $(15,16)$. Furthermore, there is no consensus with respect to the selection of an appropriate $\mathrm{PHN}$ therapy. For instance, some literatures recommended topical lidocaine as a first-line agent for PHN treatment $(17,18)$, while others denied such a recommendation due to inadequate evidence (19). Another limitation in the current literature is that the efficacy of PHN therapies was assessed using a wide range of criteria. The adoption of different criteria reduced the level of evidence consistency and caused potential contradiction.

Network meta-analysis (NMA) combines both direct and indirect evidence based on clinical trials. Nowadays, it is believed to be of high-reference-value for intervention superiority evaluation. For this sake, we designed and implemented this NMA which compared the efficacy of 9 popular PHN therapies (topical agents, antiepileptics, analgesics, antipsychotics, antidepressants, anti-dementia drugs, antivirals, A\&F, and magnesium sulfate) and came up with the optimal choice for future clinical practice.

\section{Methods}

\section{Literature Search Strategy}

Relevant studies published before August 2016, regardless of the publication date and language, were searched in China National Knowledge Infrastructure (CNKI), PubMed, and Embase using the following terms along with their synonyms: "post-herpetic neuralgia," "topical therapies," "antiepileptics," "analgesics," "opioid," "antipsychotics," "antidepressants," "anti-dementia drugs," "antivirals," "amitriptyline and fluphenazine," "magnesium sulfate," and "randomized controlled study." Additional articles from other sources, such as previous meta-analyses, were added for screening to enhance the comprehensiveness of the systematic review.

\section{Literature Screening and Inclusion}

To ensure the validity and accuracy of the systematic review, the titles and abstracts of all of the identified articles were inspected and screened independently by 2 experts, and consensus was reached through discussion when any disagreement emerged. Only those fulfilling the following criteria were included in the systematic review: 1) Studies conducted as randomized controlled trials (RCTs). 2) The data for PHN treatments could be extracted. 3) Comparison among at least 2 treatments mentioned above (placebo included) was conducted. 4) At least one pain scale from baseline to endpoint was given.

\section{Data Extraction}

Full texts of the qualified articles were accessed, and data extraction was conducted by 2 authors independently. The extracted data included the name of author, publication year, study design, follow-up period, treatment class, specific treatment, number of cases, mean age, female to male ratio, and pain scale. Any disagreement was resolved through discussion.

\section{Data Analysis}

A pain scale was the only parameter that measured the efficacy of each treatment. However, different types of pain scales were used in different included studies. Thus, we used the standardized mean difference (SMD) of pain scale changes from baseline to endpoint between each intervention group to address this issue. The results were expressed as SMD with $95 \%$ confidence intervals (Cls) for direct comparisons and credible intervals (Crls) for indirect comparisons. A negative SMD value suggested that one treatment is potentially more efficacious than another with respect to pain relief.

In the systematic review, a traditional pairwise meta-analysis was firstly performed to determine the 
relative efficacy of the different treatments for PHN. Indirect comparisons among multiple treatments were conducted by using the approach of a NMA. All statistical analyses were conducted using $R$ version 3.2.3 (The R Foundation for Statistical Computing), and the Bayesian framework was adopted for the implementation of NMA. The ranking probability of each treatment was produced by the surface under the cumulative ranking curve (SUCRA), which was established as a ranking scheme for the treatments.

Moreover, the Cochran's Q-statistic and $1^{2}$ test were evaluated to examine the heterogeneity among the selected studies, where $P<0.01$ or $\mathrm{I}^{2}>50 \%$ indicated the existence of significant heterogeneity, in which the fixed-effects model (Mantel-Haenszel method) was replaced by the random-effects model (DerSimonian-Laird method). Inconsistencies between direct and indirect evidence were also assessed on the basis of the nodesplitting method and were visualized using a net heat plot. In addition, publication bias was investigated and assessed using the funnel plot.

\section{Results}

\section{Study Characteristics}

As shown in Fig. 1, the identification, screening, and inclusion process of eligible studies is illustrated in a flowchart. Initially, 720 records were identified through initial searching in CNKI, PubMed, and Embase along with an additional 36 records added through other sources, such as references from selected meta-analyses and ClinicalTrials.gov. After removing duplicates and irrelevant articles, 321 studies remained for further inspection. Consequently, 246 records were excluded due to incomplete data or other reasons, leaving 75 records which were subject to full-text review. During data extraction, several records without sufficient data or proper comparisons that could form a network were

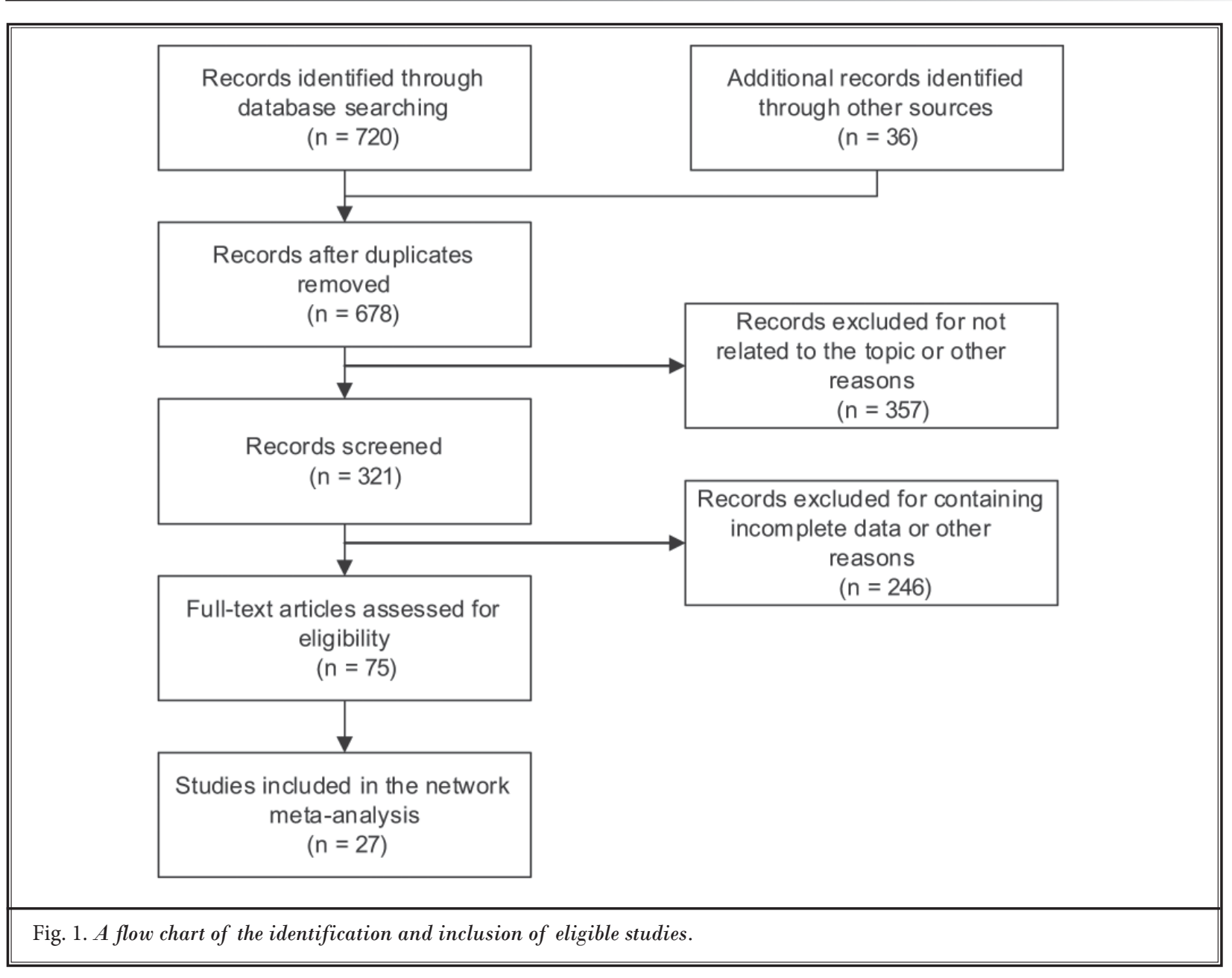

www.painphysicianjournal.com 
Pain Physician: January/February 2018; 21:19-32

further removed. As a result, 27 records were included

each enrolled study are summarized in Table $1(9,11,13-$ in this systematic review. The baseline characteristics of $16,20-40$ ). In the 27 records, 9 classes of drugs (placebo

Table 1. A description of the studies included in the Network Meta-Analysis.

\begin{tabular}{|c|c|c|c|c|c|c|c|c|c|}
\hline Author, Year & Study Design & $\begin{array}{c}\text { Follow- } \\
\text { Up } \\
\text { (wks) }\end{array}$ & $\begin{array}{l}\text { Treatment } \\
\text { Class }\end{array}$ & $\begin{array}{c}\text { Specific } \\
\text { Treatment }\end{array}$ & Cases & $\begin{array}{c}\text { Mean } \\
\text { Age } \\
(y r s)\end{array}$ & $\begin{array}{c}\text { Female } \\
(\%)\end{array}$ & $\begin{array}{l}\text { Pain } \\
\text { Scale }\end{array}$ & $\begin{array}{c}\text { Adverse } \\
\text { Event }\end{array}$ \\
\hline \multirow{2}{*}{$\begin{array}{l}\text { Surman et al } \\
(15), 1990\end{array}$} & \multirow{2}{*}{$\begin{array}{l}\text { Randomized, } \\
\text { Double-blind }\end{array}$} & 12 & Antivirals & Acyclovir & 11 & - & - & \multirow{2}{*}{ PRI } & \multirow{2}{*}{-} \\
\hline & & & Placebo & Placebo & 10 & - & - & & \\
\hline \multirow{2}{*}{$\begin{array}{l}\text { Eisenberg et al } \\
(26), 1998\end{array}$} & \multirow{2}{*}{$\begin{array}{l}\text { Randomized, } \\
\text { Double-blind }\end{array}$} & 5 & $\begin{array}{l}\text { Anti-dementia } \\
\text { drugs }\end{array}$ & Memantine & 12 & - & $58.3 \%$ & \multirow[t]{2}{*}{ NPRS } & \multirow[t]{2}{*}{$\checkmark$} \\
\hline & & & Placebo & Placebo & 12 & - & $41.7 \%$ & & \\
\hline \multirow{2}{*}{$\begin{array}{l}\text { Rowbotham et } \\
\text { al (35), } 1998\end{array}$} & \multirow{2}{*}{$\begin{array}{l}\text { Randomized, } \\
\text { Double-blind, } \\
\text { Multicenter }\end{array}$} & 8 & Antiepileptics & Gabapentin & 113 & 73.0 & $43.1 \%$ & \multirow{2}{*}{$\begin{array}{l}\text { Likert } \\
\text { Scale }\end{array}$} & \multirow{2}{*}{$\checkmark$} \\
\hline & & & Placebo & Placebo & 116 & 74.0 & $51.7 \%$ & & \\
\hline \multirow{4}{*}{$\begin{array}{l}\text { Graff-Radford } \\
\text { et al (11), } 2000\end{array}$} & \multirow{4}{*}{ Double-blind } & 8 & Antidepressants & Amitriptyline & 11 & 76.5 & $27.3 \%$ & \multirow{4}{*}{ VAS } & \multirow{4}{*}{ - } \\
\hline & & & $\mathrm{A} \& \mathrm{~F}$ & $\mathrm{~A} \& \mathrm{~F}$ & 12 & 70.2 & $50.0 \%$ & & \\
\hline & & & Antipsychotics & Fluphenazine & 13 & 71.5 & $53.8 \%$ & & \\
\hline & & & Placebo & Placebo & 13 & 73.9 & $53.8 \%$ & & \\
\hline \multirow{2}{*}{$\begin{array}{l}\text { Rice et al (34), } \\
2001\end{array}$} & \multirow{2}{*}{$\begin{array}{l}\text { Randomized, } \\
\text { Double-blind }\end{array}$} & 7 & Antiepileptics & Gabapentin & 223 & 75.5 & $58.7 \%$ & \multirow{2}{*}{ VAS } & \multirow{2}{*}{$\checkmark$} \\
\hline & & & Placebo & Placebo & 111 & 74.9 & $58.6 \%$ & & \\
\hline \multirow{2}{*}{$\begin{array}{l}\text { Galer et al (27), } \\
2002\end{array}$} & \multirow{2}{*}{$\begin{array}{l}\text { Randomized, } \\
\text { Double-blind }\end{array}$} & 3 & Topical therapies & Lidocaine & 67 & 74.0 & $62.7 \%$ & \multirow{2}{*}{ NPS } & \multirow{2}{*}{-} \\
\hline & & & Placebo & Placebo & 29 & 74.0 & $62.1 \%$ & & \\
\hline \multirow{3}{*}{$\begin{array}{l}\text { Raja et al (32), } \\
2002\end{array}$} & \multirow{3}{*}{ Randomized } & 24 & Analgesics & Opioid & 71 & - & - & & \\
\hline & & & Antidepressants & TCA & 60 & - & - & NRS & $\checkmark$ \\
\hline & & & Placebo & Placebo & 50 & - & - & & \\
\hline Boureau et al & $\begin{array}{l}\text { Randomized, } \\
\text { Double-blind, }\end{array}$ & 6 & Analgesics & $\begin{array}{c}\text { Tramadol } \\
\text { hydrochloride }\end{array}$ & 64 & 65.7 & $62.3 \%$ & VAS & $\checkmark$ \\
\hline$(23), 2003$ & Parallel-group & & Placebo & Placebo & 63 & 67.9 & $80.0 \%$ & & \\
\hline Dworkin et al & Dondomind & 8 & Antiepileptics & Pregabalin & 89 & 72.4 & $58.4 \%$ & DI NDC & 1 \\
\hline$(25), 2003$ & Kancominzed & & Placebo & Placebo & 84 & 70.5 & $47.6 \%$ & PI-NKS & 年 \\
\hline Sabatowski et al & Pondlomiza & 8 & Antiepileptics & Pregabalin & 157 & 71.6 & $55.4 \%$ & VAS & 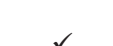 \\
\hline (36), 2004 & Randomized & & Placebo & Placebo & 81 & 73.2 & $54.3 \%$ & VAS & $v$ \\
\hline Kochar et al & Randomized, & 8 & Antiepileptics & $\begin{array}{c}\text { Divalproex } \\
\text { sodium }\end{array}$ & 23 & 58.0 & $45.5 \%$ & PPI & - \\
\hline & & & Placebo & Placebo & 22 & 56.4 & $44.4 \%$ & & \\
\hline Chandra, et al & Randomized, & 8 & Antidepressants & Nortriptyline & 36 & 52.5 & $61.2 \%$ & & \\
\hline (24) 2006 & $\begin{array}{l}\text { Double-bind, } \\
\text { Parallel-group }\end{array}$ & & Antiepileptics & Gabapentin & 34 & 55.6 & $41.2 \%$ & VAS & - \\
\hline & Randomized, & 13 & Antiepileptics & Pregabalin & 275 & 70.6 & $53.5 \%$ & & \\
\hline $\begin{array}{l}\text { van Seventer et } \\
\text { al (37), } 2006\end{array}$ & $\begin{array}{l}\text { Double-blind, } \\
\text { Multicenter, } \\
\text { Parallel-group }\end{array}$ & & Placebo & Placebo & 93 & 70.9 & $53.0 \%$ & NRS & $\checkmark$ \\
\hline Backonja et al & Randomized, & 12 & Topical therapies & NGX-4010 & 206 & 71.5 & $52.0 \%$ & NIDPC & 1 \\
\hline (9), 2008 & Double-bind & & Placebo & Placebo & 196 & 70.7 & $53.0 \%$ & NPRS & $v$ \\
\hline Baron et al (29), & Randomized, Open- & 12 & Topical therapies & Lidocaine & 45 & 66.0 & $48.9 \%$ & NRS & $\checkmark$ \\
\hline 2009 & label, Multicenter & & Antiepileptics & Pregabalin & 43 & 63.8 & $51.2 \%$ & NRS & $v$ \\
\hline Irving et al (29), & Randomized, & 4 & Antiepileptics & Gabapentin & 107 & 69.5 & $54.2 \%$ & NDP & 4 \\
\hline 2009 & Double-blind & & Placebo & Placebo & 51 & 69.0 & $51.0 \%$ & NKS & v \\
\hline
\end{tabular}


Pain Relief in PHN Therapies

Table 1 con't. A description of the studies included in the NMA.

\begin{tabular}{|c|c|c|c|c|c|c|c|c|c|}
\hline Author, Year & Study Design & $\begin{array}{c}\text { Follow- } \\
\text { Up } \\
\text { (wks) }\end{array}$ & $\begin{array}{l}\text { Treatment } \\
\text { Class }\end{array}$ & $\begin{array}{c}\text { Specific } \\
\text { Treatment }\end{array}$ & Cases & $\begin{array}{c}\text { Mean } \\
\text { Age } \\
\text { (yrs) }\end{array}$ & $\begin{array}{c}\text { Female } \\
(\%)\end{array}$ & $\begin{array}{l}\text { Pain } \\
\text { Scale }\end{array}$ & $\begin{array}{c}\text { Adverse } \\
\text { Event }\end{array}$ \\
\hline \multirow{2}{*}{$\begin{array}{l}\text { Jensen et al } \\
(30), 2009\end{array}$} & \multirow{2}{*}{ Randomized } & 2 & Antiepileptics & Gabapentin & 102 & 69.5 & $52.9 \%$ & \multirow{2}{*}{ NRS } & \multirow{2}{*}{ - } \\
\hline & & & Placebo & Placebo & 49 & 70.0 & $51.0 \%$ & & \\
\hline \multirow{2}{*}{$\begin{array}{l}\text { Backonja et al } \\
(21), 2010\end{array}$} & \multirow{2}{*}{$\begin{array}{l}\text { Randomized, } \\
\text { Double-Blind, } \\
\text { Controlled Study }\end{array}$} & 4 & $\begin{array}{l}\text { Topical } \\
\text { therapies }\end{array}$ & NGX-4010 & 26 & 74.4 & $77.0 \%$ & \multirow[t]{2}{*}{ NPRS } & \multirow[t]{2}{*}{$\checkmark$} \\
\hline & & & Placebo & Placebo & 12 & 76.0 & $25.0 \%$ & & \\
\hline \multirow{2}{*}{$\begin{array}{l}\text { Rehm et al (33), } \\
2010\end{array}$} & \multirow{2}{*}{ Randomized } & 4 & Topical therapies & Lidocaine & 50 & - & - & \multirow{2}{*}{ NPSI } & \multirow{2}{*}{$\checkmark$} \\
\hline & & & Antiepileptics & Pregabalin & 48 & - & - & & \\
\hline \multirow{2}{*}{$\begin{array}{l}\text { Wallace et al } \\
(38), 2010\end{array}$} & \multirow{2}{*}{$\begin{array}{l}\text { Randomized, } \\
\text { Double-blind, } \\
\text { Multicenter }\end{array}$} & 10 & Antiepileptics & Gabapentin & 269 & 67.1 & $52.7 \%$ & \multirow{2}{*}{$\mathrm{ADP}$} & \multirow{2}{*}{$\checkmark$} \\
\hline & & & Placebo & Placebo & 131 & 66.0 & $41.0 \%$ & & \\
\hline \multirow{2}{*}{$\begin{array}{l}\text { Webster et al } \\
(39), 2010\end{array}$} & \multirow{2}{*}{$\begin{array}{l}\text { Randomized, } \\
\text { Multicenter }\end{array}$} & 12 & Topical therapies & NGX-4010 & 102 & 68.7 & $54.0 \%$ & \multirow{2}{*}{ NPRS } & \multirow{2}{*}{$\checkmark$} \\
\hline & & & Placebo & Placebo & 53 & 71.2 & $53.0 \%$ & & \\
\hline \multirow{2}{*}{$\begin{array}{l}\text { Webster et al } \\
(40), 2010\end{array}$} & \multirow{2}{*}{$\begin{array}{l}\text { Randomized, } \\
\text { Double-Blind, } \\
\text { Multicenter }\end{array}$} & 12 & Topical therapies & NGX-4010 & 222 & 71.7 & $49.4 \%$ & \multirow[b]{2}{*}{ NPRS } & \multirow[b]{2}{*}{$\checkmark$} \\
\hline & & & Placebo & Placebo & 77 & 71.1 & $51.0 \%$ & & \\
\hline \multirow{2}{*}{$\begin{array}{l}\text { Backonja et al } \\
(20), 2011\end{array}$} & \multirow{2}{*}{$\begin{array}{l}\text { Randomized, } \\
\text { Double-Blind }\end{array}$} & 2 & Antiepileptics & Gabapentin & 48 & 65.0 & $53.2 \%$ & \multirow{2}{*}{ NPS } & \multirow{2}{*}{$\checkmark$} \\
\hline & & & Placebo & Placebo & 54 & 64.0 & $50.0 \%$ & & \\
\hline \multirow{2}{*}{$\begin{array}{l}\text { Irving et al (29), } \\
2011\end{array}$} & \multirow{2}{*}{$\begin{array}{c}\text { Randomized, } \\
\text { Double-Blind, } \\
\text { Multicenter }\end{array}$} & 12 & Topical therapies & NGX-4010 & 212 & 70.2 & $56.0 \%$ & \multirow{2}{*}{ NPRS } & \multirow{2}{*}{$\checkmark$} \\
\hline & & & Placebo & Placebo & 204 & 70.4 & $52.0 \%$ & & \\
\hline \multirow{2}{*}{$\begin{array}{l}\text { Smith et al (14), } \\
2014\end{array}$} & Randomized, & 8 & Antiepileptics & Carisbamate & 75 & 65.0 & $62.7 \%$ & בסני & 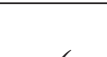 \\
\hline & $\begin{array}{l}\text { Double-blind, } \\
\text { Proof-of-concept }\end{array}$ & & Placebo & Placebo & 75 & 65.0 & $62.7 \%$ & NRS & $\checkmark$ \\
\hline Liu et al (13), & Randomized, & 8 & Antiepileptics & Pregabalin & 111 & 65.7 & $48.6 \%$ & DPRS & 1 \\
\hline 2017 & Double-blind & & Placebo & Placebo & 109 & 64.1 & $43.1 \%$ & DPRS & \\
\hline Kim et al (16), & Randomized, & 2 & Analgesics & $\begin{array}{c}\text { Ketamine } \\
\text { hydrochloride }\end{array}$ & 15 & - & - & Us & . \\
\hline 2015 & Double-blind & & $\begin{array}{l}\text { Magnesium } \\
\text { sulfate }\end{array}$ & $\begin{array}{l}\text { Magnesium } \\
\text { sulfate }\end{array}$ & 15 & - & - & VAS & - \\
\hline
\end{tabular}

Abbreviations: $\mathrm{A} \& \mathrm{~F}=$ amitriptyline and fluphenazine; $\mathrm{TCA}=$ tricyclic antidepressants; $\mathrm{PRI}$ = pain rating index; NPRS = numeric pain rating scale VAS = visual analog scale for pain; NPS = neuropathic pain scale; NRS = numeric rating scale; PI-NRS = pain intensity numerical rating scale; PPI = present pain intensity score; NPSI = neuropathic pain symptom inventory; $\mathrm{ADP}=$ average daily pain score; $\mathrm{DPRS}=$ daily pain rating scale; $-=$ not specified

not included) were involved: topical therapies, antiepileptics, analgesics, antipsychotics, antidepressants, antidementia drugs, antivirals, A\&F, and magnesium sulfate. As illustrated in Fig. 2, the corresponding direct and indirect comparisons are presented in the network plot.

\section{Risk of Publication Bias}

The effect of study sizes versus standard errors was investigated via funnel plots. Typically, publication bias, which implies differences among different study sizes, is reported to show if an asymmetrical funnel plot occurs. As shown in Fig. S1, the funnel plot pattern in the systematic review exhibited no significant asymmetry pattern, indicating a low risk of publication bias.

\section{Pairwise Comparison}

As shown in Table 2, a total of 15 groups of traditional pairwise comparison were conducted, in which SMD, 95\% Cls, and $P$-value of each comparison were calculated and presented. In contrast to placebo, 4 treatment classes, including topical therapies (SMD $=-0.23$; 95\% Cl: $-0.36,-0.09$ ), antiepileptics (SMD $=-0.72 ; 95 \% \mathrm{Cl}$ : $-1.06,-0.38)$, analgesics (SMD $=-0.64 ; 95 \% \mathrm{Cl}:-1.13,-0.15)$, and antidepressants (SMD $=-0.70 ; 95 \% \mathrm{Cl}:-1.36,-0.04)$, 


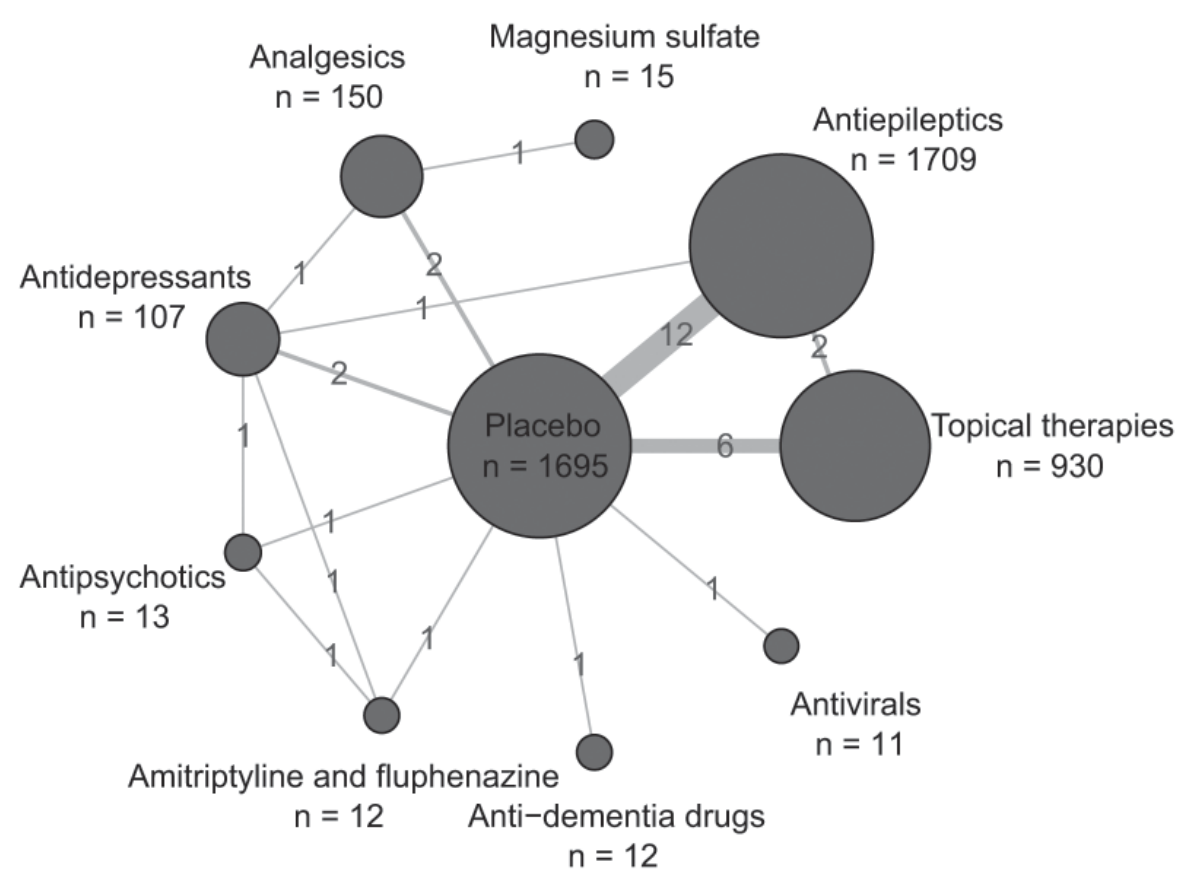

Fig. 2. A network of comparisons between the different classes of treatments in this NMA. The size of a circle indicates the number of cases of the treatment class and the thickness of the line between 2 circles indicates the number of studies comparing the 2 treatment classes.

Table 2. The direct comparisons of the different classes of treatments.

\begin{tabular}{|c|c|c|c|c|c|}
\hline \multirow{2}{*}{ Class 1 vs. Class 2} & \multirow{2}{*}{ SMD $^{\mathbf{a}}$} & \multicolumn{2}{|c|}{ 95\% CI Limits } & \multirow{2}{*}{$P$-value ${ }^{\mathrm{b}}$} & \multirow{2}{*}{ Significant $^{\mathrm{c}}$} \\
\hline & & Lower & Upper & & \\
\hline Topical therapies vs. Placebo & -0.23 & -0.36 & -0.09 & 0.001 & $\checkmark$ \\
\hline Antiepileptics vs. Placebo & -0.72 & -1.06 & -0.38 & 0.000 & $\checkmark$ \\
\hline Antiepileptics vs. Topical therapies & 0.15 & -0.14 & 0.43 & 0.319 & \\
\hline Analgesics vs. Placebo & -0.64 & -1.13 & -0.15 & 0.010 & $\checkmark$ \\
\hline Antipsychotics vs. Placebo & -0.26 & -1.04 & 0.51 & 0.504 & \\
\hline Antidepressants vs. Placebo & -0.70 & -1.36 & -0.04 & 0.039 & $\checkmark$ \\
\hline Antidepressants vs. Antiepileptics & -0.18 & -0.65 & 0.29 & 0.465 & \\
\hline Antidepressants vs. Analgesics & 0.40 & 0.05 & 0.75 & 0.025 & $\checkmark$ \\
\hline Antidepressants vs. Antipsychotics & -0.82 & -1.66 & 0.02 & 0.056 & \\
\hline Anti-dementia drugs vs. Placebo & -0.19 & -0.99 & 0.62 & 0.650 & \\
\hline Antivirals vs. Placebo & 0.60 & -0.28 & 1.48 & 0.182 & \\
\hline A\&F vs. Placebo & -0.31 & -1.10 & 0.48 & 0.437 & \\
\hline A\&F vs. Antipsychotics & -0.03 & -0.82 & 0.76 & 0.940 & \\
\hline A\&F vs. Antidepressants & 0.86 & 0.00 & 1.72 & 0.050 & $\checkmark$ \\
\hline Magnesium sulfate vs. Analgesics & -0.13 & -0.85 & 0.59 & 0.726 & $\checkmark$ \\
\hline
\end{tabular}

Abbreviations: $\mathrm{SMD}=$ standardized mean difference; $\mathrm{A} \& \mathrm{~F}=$ amitriptyline and fluphenazine. ${ }^{\text {aNegative }}$ SMDs indicate that efficacy of class one is better than class $2 .{ }^{\mathrm{b}} \mathrm{P}<0.01$ indicated the significant heterogeneity. ${ }^{\mathrm{C}} \mathrm{S} M D$ s that are statistically significant are marked by ticks. 
Pain Relief in PHN Therapies

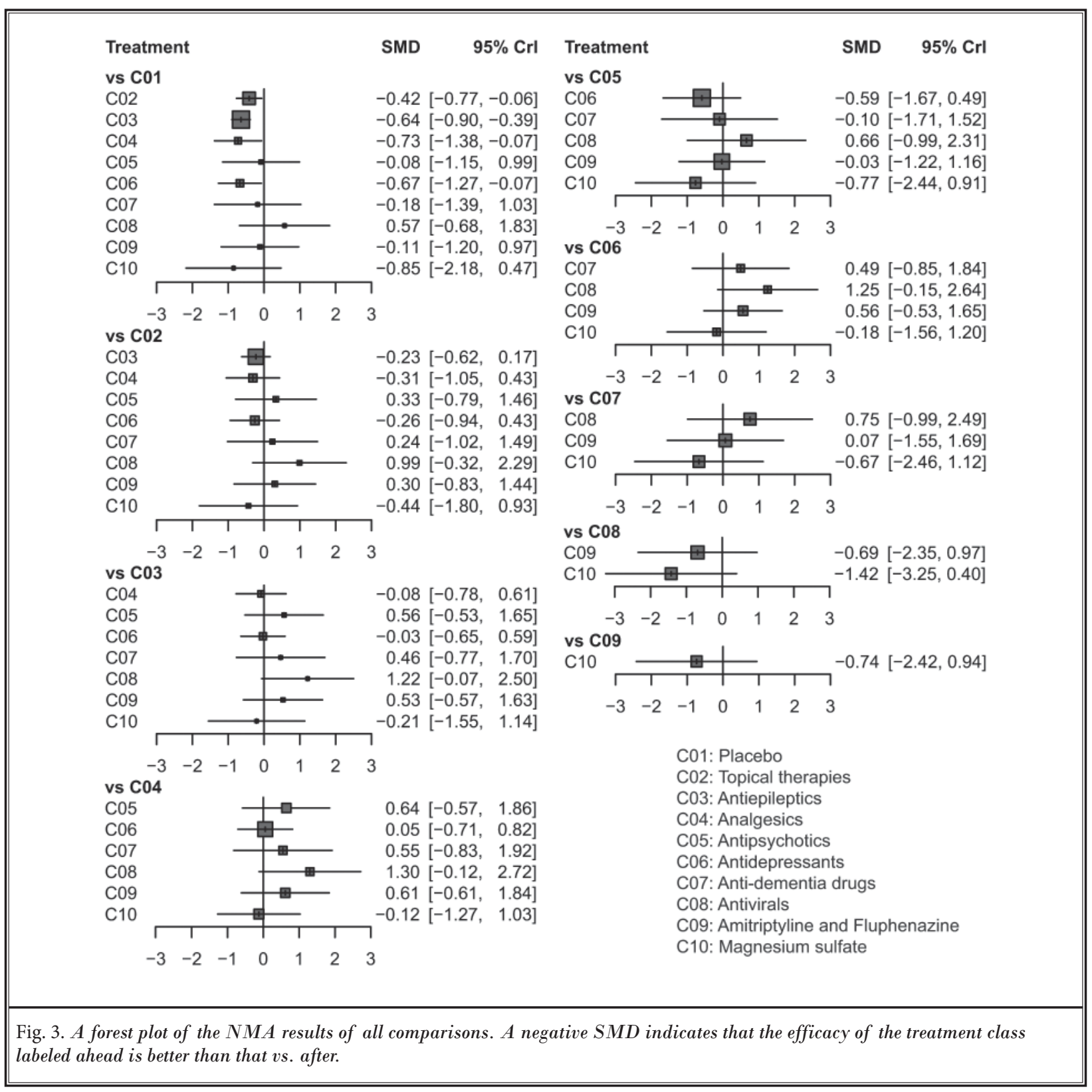

exhibited a significant pain relief effect. Moreover, analgesics showed significantly superior efficacy compared to antidepressants (antidepressants vs. analgesics SMD = $0.40 ; 95 \% \mathrm{Cl}: 0.05,0.75)$, while A\&F was significantly less effective than antidepressants (A\&F vs. antidepressants SMD $=0.86 ; 95 \% \mathrm{Cl}: 0.01,0.72$ ).

\section{NMA}

Results of the NMA are displayed in Table 3 and Fig. 3. Patients with 4 treatment classes exhibited significant improvements in pain scores compared to those with placebo: antiepileptics (SMD $=-0.64 ; 95 \% \mathrm{Crl}$ : -0.90 , -0.39), analgesics (SMD $=-0.73 ; 95 \% \mathrm{Crl}:-1.38,-0.07$ ), antidepressants (SMD $=-0.67 ; 95 \% \mathrm{Crl}:-1.27,-0.07$ ), and topical therapies (SMD $=-0.42 ; 95 \% \mathrm{Crl}:-0.77,-0.06)$.

\section{Consistency Assessment}

This systematic review was based on a consistency model, the validity of which could be compromised by significant inconsistency between direct and indirect 
Pain Physician: January/February 2018; 21:19-32

Table 3. The NMA of the different classes of treatments.

\begin{tabular}{|c|c|c|c|c|c|c|c|c|c|}
\hline $\mathbf{A}$ & $\begin{array}{c}0.42 \\
(0.06 \\
0.77)\end{array}$ & $\begin{array}{c}0.64 \\
(0.39 \\
0.90)\end{array}$ & $\begin{array}{c}0.73 \\
(0.07 \\
1.38)\end{array}$ & $\begin{array}{c}0.08 \\
(-0.99 \\
1.15)\end{array}$ & $\begin{array}{c}0.67 \\
(0.07 \\
1.27)\end{array}$ & $\begin{array}{c}0.18 \\
(-1.03 \\
1.39)\end{array}$ & $\begin{array}{c}-\mathbf{- 0 . 5 7} \\
(-1.83 \\
0.68)\end{array}$ & $\begin{array}{c}0.11 \\
(-0.97 \\
1.20)\end{array}$ & $\begin{array}{c}\mathbf{0 . 8 5} \\
(-0.47 \\
2.18)\end{array}$ \\
\hline $\begin{array}{c}-0.42 \\
(-0.77,-0.06)\end{array}$ & B & $\begin{array}{c}0.23 \\
(-0.17,0.62)\end{array}$ & $\begin{array}{c}0.31 \\
(-0.43,1.05)\end{array}$ & $\begin{array}{c}-0.33 \\
(-1.46,0.79)\end{array}$ & $\begin{array}{c}0.26 \\
(-0.43,0.94)\end{array}$ & $\begin{array}{c}-0.24 \\
(-1.49,1.02)\end{array}$ & $\begin{array}{c}-0.99 \\
(-2.29,0.32)\end{array}$ & $\begin{array}{c}-0.30 \\
(-1.44,0.83)\end{array}$ & $\begin{array}{c}0.44 \\
(-0.93,1.80)\end{array}$ \\
\hline $\begin{array}{c}-0.64 \\
(-0.90,-0.39)\end{array}$ & $\begin{array}{c}-0.23 \\
(-0.62,0.17)\end{array}$ & C & $\begin{array}{c}0.08 \\
(-0.61,0.78)\end{array}$ & $\begin{array}{c}-0.56 \\
(-1.65,0.53)\end{array}$ & $\begin{array}{c}0.03 \\
(-0.59,0.65)\end{array}$ & $\begin{array}{c}-0.46 \\
(-1.7,0.77) \\
\end{array}$ & $\begin{array}{c}-1.22 \\
(-2.50,0.07)\end{array}$ & $\begin{array}{c}-0.53 \\
(-1.63,0.57)\end{array}$ & $\begin{array}{c}0.21 \\
(-1.14,1.55)\end{array}$ \\
\hline $\begin{array}{c}-0.73 \\
(-1.38,-0.07)\end{array}$ & $\begin{array}{c}-0.31 \\
(-1.05,0.43)\end{array}$ & $\begin{array}{c}-0.08 \\
(-0.78,0.61)\end{array}$ & $\mathrm{D}$ & $\begin{array}{c}-0.64 \\
(-1.86,0.57)\end{array}$ & $\begin{array}{c}-0.05 \\
(-0.82,0.71)\end{array}$ & $\begin{array}{c}-0.55 \\
(-1.92,0.83)\end{array}$ & $\begin{array}{c}-1.30 \\
(-2.72,0.12)\end{array}$ & $\begin{array}{c}-0.61 \\
(-1.84,0.61)\end{array}$ & $\begin{array}{c}0.12 \\
(-1.03,1.27)\end{array}$ \\
\hline $\begin{array}{c}-0.08 \\
(-1.15,0.99)\end{array}$ & $\begin{array}{c}0.33 \\
(-0.79,1.46)\end{array}$ & $\begin{array}{c}0.56 \\
(-0.53,1.65)\end{array}$ & $\begin{array}{c}0.64 \\
(-0.57,1.86)\end{array}$ & $\mathrm{E}$ & $\begin{array}{c}0.59 \\
(-0.49,1.67)\end{array}$ & $\begin{array}{c}0.10 \\
(-1.52,1.71)\end{array}$ & $\begin{array}{c}-0.66 \\
(-2.31,0.99)\end{array}$ & $\begin{array}{c}0.03 \\
(-1.16,1.22)\end{array}$ & $\begin{array}{c}0.77 \\
(-0.91,2.44)\end{array}$ \\
\hline $\begin{array}{c}-0.67 \\
(-1.27,-0.07)\end{array}$ & $\begin{array}{c}-0.26 \\
(-0.94,0.43)\end{array}$ & $\begin{array}{c}-0.03 \\
(-0.65,0.59)\end{array}$ & $\begin{array}{c}0.05 \\
(-0.71,0.82)\end{array}$ & $\begin{array}{c}-0.59 \\
(-1.67,0.49)\end{array}$ & $\mathrm{F}$ & $\begin{array}{c}-0.49 \\
(-1.84,0.85)\end{array}$ & $\begin{array}{c}-1.25 \\
(-2.64,0.15)\end{array}$ & $\begin{array}{c}-0.56 \\
(-1.65,0.53)\end{array}$ & $\begin{array}{c}0.18 \\
(-1.20,1.56)\end{array}$ \\
\hline $\begin{array}{c}-0.18 \\
(-1.39,1.03)\end{array}$ & $\begin{array}{c}0.24 \\
(-1.02,1.49)\end{array}$ & $\begin{array}{c}0.46 \\
(-0.77,1.70)\end{array}$ & $\begin{array}{c}0.55 \\
(-0.83,1.92)\end{array}$ & $\begin{array}{c}-0.10 \\
(-1.71,1.52)\end{array}$ & $\begin{array}{c}0.49 \\
(-0.85,1.84)\end{array}$ & $\mathrm{G}$ & $\begin{array}{c}-0.75 \\
(-2.49,0.99)\end{array}$ & $\begin{array}{c}-0.07 \\
(-1.69,1.55)\end{array}$ & $\begin{array}{c}0.67 \\
(-1.12,2.46)\end{array}$ \\
\hline $\begin{array}{c}0.57 \\
(-0.68,1.83) \\
\end{array}$ & $\begin{array}{c}0.99 \\
(-0.32,2.29) \\
\end{array}$ & $\begin{array}{c}1.22 \\
(-0.07,2.50) \\
\end{array}$ & $\begin{array}{c}1.30 \\
(-0.12,2.72) \\
\end{array}$ & $\begin{array}{c}0.66 \\
(-0.99,2.31) \\
\end{array}$ & $\begin{array}{c}1.25 \\
(-0.15,2.64) \\
\end{array}$ & $\begin{array}{c}0.75 \\
(-0.99,2.49) \\
\end{array}$ & $\mathrm{H}$ & $\begin{array}{c}0.69 \\
(-0.97,2.35)\end{array}$ & $\begin{array}{c}1.42 \\
(-0.40,3.25)\end{array}$ \\
\hline $\begin{array}{c}-0.11 \\
(-1.20,0.97) \\
\end{array}$ & $\begin{array}{c}0.30 \\
(-0.83,1.44) \\
\end{array}$ & $\begin{array}{c}0.53 \\
(-0.57,1.63) \\
\end{array}$ & $\begin{array}{c}0.61 \\
(-0.61,1.84) \\
\end{array}$ & $\begin{array}{c}-0.03 \\
(-1.22,1.16)\end{array}$ & $\begin{array}{c}0.56 \\
(-0.53,1.65) \\
\end{array}$ & $\begin{array}{c}0.07 \\
(-1.55,1.69) \\
\end{array}$ & $\begin{array}{c}-0.69 \\
(-2.35,0.97)\end{array}$ & I & $\begin{array}{c}0.74 \\
(-0.94,2.42) \\
\end{array}$ \\
\hline $\begin{array}{c}-0.85 \\
(-2.18,0.47)\end{array}$ & $\begin{array}{c}-0.44 \\
(-1.8,0.93)\end{array}$ & $\begin{array}{c}-0.21 \\
(-1.55,1.14)\end{array}$ & $\begin{array}{c}-0.12 \\
(-1.27,1.03)\end{array}$ & $\begin{array}{c}-0.77 \\
(-2.44,0.91)\end{array}$ & $\begin{array}{c}-0.18 \\
(-1.56,1.2)\end{array}$ & $\begin{array}{c}-0.67 \\
(-2.46,1.12)\end{array}$ & $\begin{array}{c}-1.42 \\
(-3.25,0.40)\end{array}$ & $\begin{array}{c}-0.74 \\
(-2.42,0.94)\end{array}$ & $\mathrm{J}$ \\
\hline
\end{tabular}

Abbreviations: $\mathrm{A}=$ placebo; $\mathrm{B}=$ topical therapies; $\mathrm{C}=$ antiepileptics; $\mathrm{D}=$ analgesics; $\mathrm{E}=$ antipsychotics; $\mathrm{F}=$ antidepressants; $\mathrm{G}=$ anti-dementia drugs; $\mathrm{H}=$ antivirals; $\mathrm{I}=\mathrm{A} \& \mathrm{~F} ; \mathrm{J}=$ magnesium sulfate

A value in the matrix indicates the difference between the standardized mean value of the treatment in the row and in the column. The value is arranged in the form of SMD (95\% CI lower limit, 95\% CI upper limit).

SMDs that are statistically significant are emphasized in boldface.

evidence. The node-splitting method (41) was employed to assess the degree of consistency in the systematic review, where $P<0.01$ implied significant inconsistency. The results are shown in Table S1, suggesting that there was no significant inconsistency between direct and indirect evidence in the systematic review. Additionally, a net heat plot was drawn in Fig. S2 (42). Similarly, no significant inconsistency between direct and indirect comparisons was observed.

\section{Ranking of Treatment Classes}

As shown in Fig. 4, the rank probabilities of each treatment class were calculated and cumulative probability rank curves were plotted. The mean rank and SUCRA of each treatment were also calculated and are presented in Table S2. Analgesics and magnesium sulfate ranked first and second concerning pain relief, respectively and were considered to be optimal candidates for PHN treatment with SUCRAs over 0.70. The secondary favorable treatments were antiepileptics and antidepressants, both of which had a SUCRA value around 0.60 . In contrast, antivirals appeared to be the least favorable treatment due to having the lowest SUCRA values.

\section{Discussion}

In this NMA, we focused on the comparable efficacy of 9 classes of PHN therapies. In view of pain relief or pain intensity measured using different scales (pain rating index [PRI], numeric pain rating scale [NPRS], visual analog scale [VAS]) in the included studies, we evaluated the mean changes on pain intensity before and after the treatments and compared the pain relief effects using the statistics of SMD. In this NMA, both direct and indirect evidence demonstrated that analgesics, antidepressants, antiepileptics, and topical therapies were significantly preferable than placebo with respect to pain relief, which is consistent to the recommendation of previous guidelines (43). As for the overall rank, it was suggested by our SUCRA results that analgesics and magnesium sulfate exhibited the best efficacy with respect to PHN pain relief, whereas antivirals appeared to be the least effective class of therapy.

Consistent to our results, the pain relief effect of analgesics and magnesium sulfate have been found and verified for years in several neuropathic pains, such as migraine pain, phantom limb pain, and nerve injury pain (44-46). The underlying mechanisms linking these 2 therapies to PHN pain relief may be associated 
with the N-methyl-D-aspartate (NMDA) receptor. The NMDA receptor, which is reported to be involved in the control of PHN, is of great importance to the development of central sensitization, neuroplasticity changes, and expansion of receptive fields in the central nervous system (47-49). Both ketamine (a type of analgesics) and magnesium sulfate are NMDA antagonists, and by blocking the phencyclidine site and calcium channels of the NMDA receptor, respectively, these 2 therapies can arrest the neural activity mentioned above, thus suppressing neuropathic pain $(47,50)$.

However, the direct evidence for magnesium sulfate was based on only one study with 15 cases, which may make the results biased. This limitation also existed in the evaluation of some other effective therapies in this systematic review, including analgesics and antidepressants. For this reason, we noticed another class of PHN therapy, antiepileptics, which was well-supported by sufficient evidence in this systematic review and exhibited its efficacy on pain relief.

Numerous studies have focused on the pain relief effect of antiepileptics for patients with PHN. For instance, gabapentinoid antiepileptic drugs have exhibited prominent efficacy on reducing PHN pain in a variety of clinical trials $(28,35)$ and were recommended as the first-line treatments for PHN (51). The pharmacological mechanism lies in that gabapentinoid drugs can inhibit calcium currents by high-voltage-activated channels, contributing to the reduction of neurotransmitter release and attenuation of postsynaptic excitability. However, carisbamate, a novel antiepileptic, did not outperform placebo in a study included in our NMA (14), suggesting that not all of the antiepileptics for PHN pain relief were effective.

Additionally, the efficacy of antivirals ranked the lowest and even less effective than placebo. Antiviral agents are not the treatments for PHN pain relief, but are interventions for PHN prevention (52). Besides that, there is only one direct comparison containing antivirals in this systematic review, and only one kind of antiviral agent was assessed (15). Moreover, acyclovir, the only antiviral agent evaluated in this systematic review, has been confirmed ineffective in reducing the incidence of $\mathrm{PHN}$ in previous literatures $(52,53)$. However, evidence for other new antiviral agents was hard to find, and therefore the effect of antivirals on PHN prevention or pain relief is still in need of future investigations.

There are several limitations of this systematic review. Firstly, as mentioned above, there is a lack of direct head-to-head comparisons of some treatments, especially for antivirals, anti-dementia drugs, and magnesium sulfate. Though this limitation was addressed by the method of NMA to a certain extent, more direct evidence is still needed to draw more robust and reliable conclusions. Secondly, the specific agents belonging to a same class of therapies might exhibit different effects (gabapentin and carisbamate) with different mechanisms (opioids and ketamine) on reducing pain, and some agents were hard to find in literatures and were not involved in the systematic review, which may influence our results and contribute to the heterogeneity. In addition, some confounding factors which were inherent in the studies we included, such as the age of patients and the dose of agents, can also affect the results of this systematic review. Moreover, this NMA only focused on the pain relief effect of these treatments and did not assess the adverse events. Therefore, more well-designed studies, which take these confounding factors into consideration and measure the adverse events, are needed to improve the analysis.

In conclusion, both of the NMDA receptor antagonists, analgesics and magnesium sulfate, were preferable to the other treatments with respect to pain relief for PHN, although further evidence were required to confirm their clinical performance, while antivirals was the least effective class and even worse than placebo and should be ruled out when carrying out clinical practice based on our analysis.

\section{Author's Contributions}

DS, $A H$, and $R X$ were responsible for the literature search, data extraction, and manuscript writing; $\mathrm{AH}$ and $\mathrm{XX}$ also contributed to the literature search and data extraction; $D S$ and $R X$ were responsible for the statistical analysis; $A H, X X$, and $Y W$ contributed to the revision of the manuscript and experimental design; YW is responsible for the overall content as the guarantor. All of the authors have read and approved the final manuscript. 

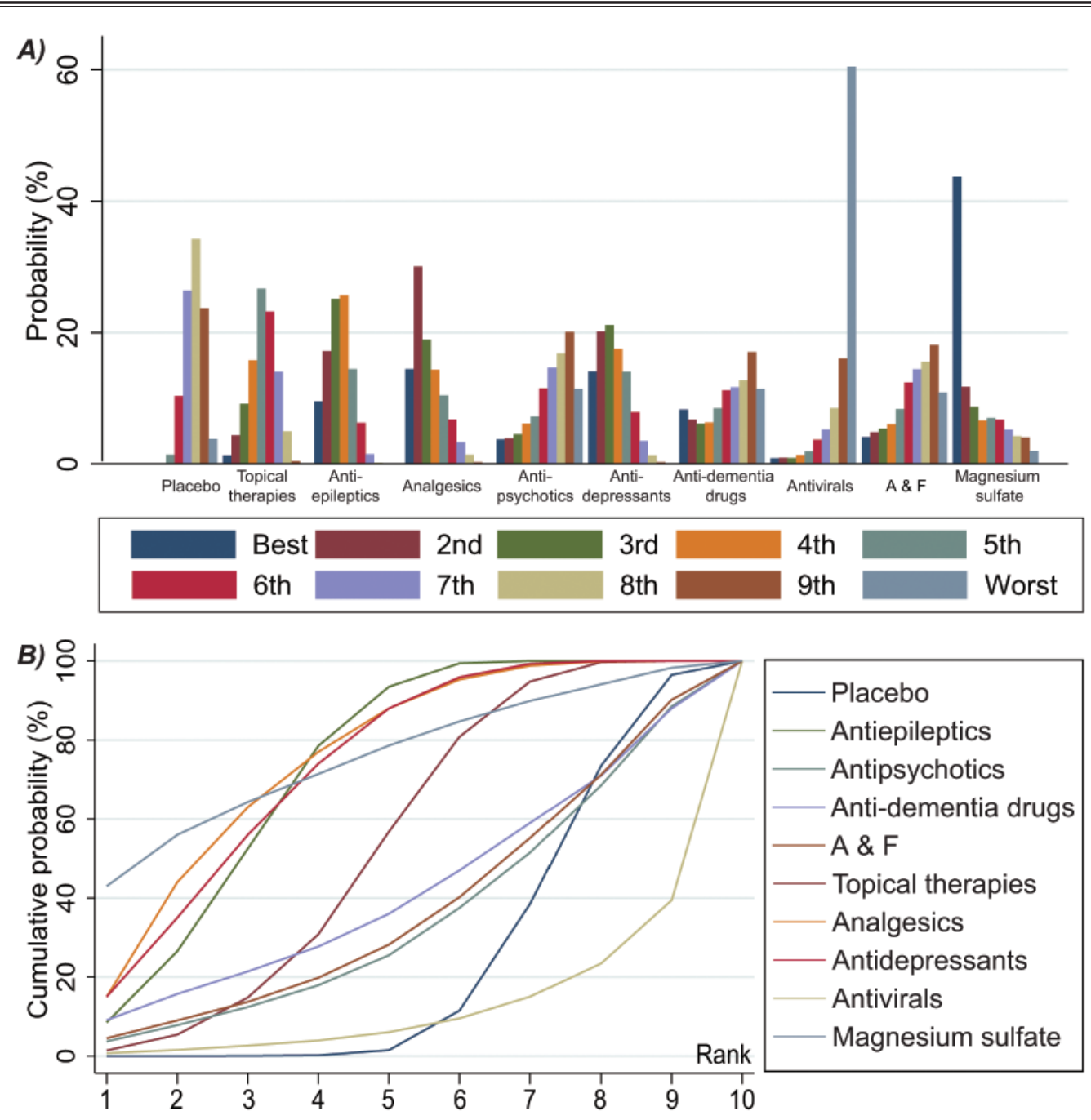

Fig. 4. Diagrams of rank analysis of treatment classes. A) A bar plot of the probability of each treatment class in each specific rank. B) A line plot of the cumulative rank probabilities of all of the treatment classes.

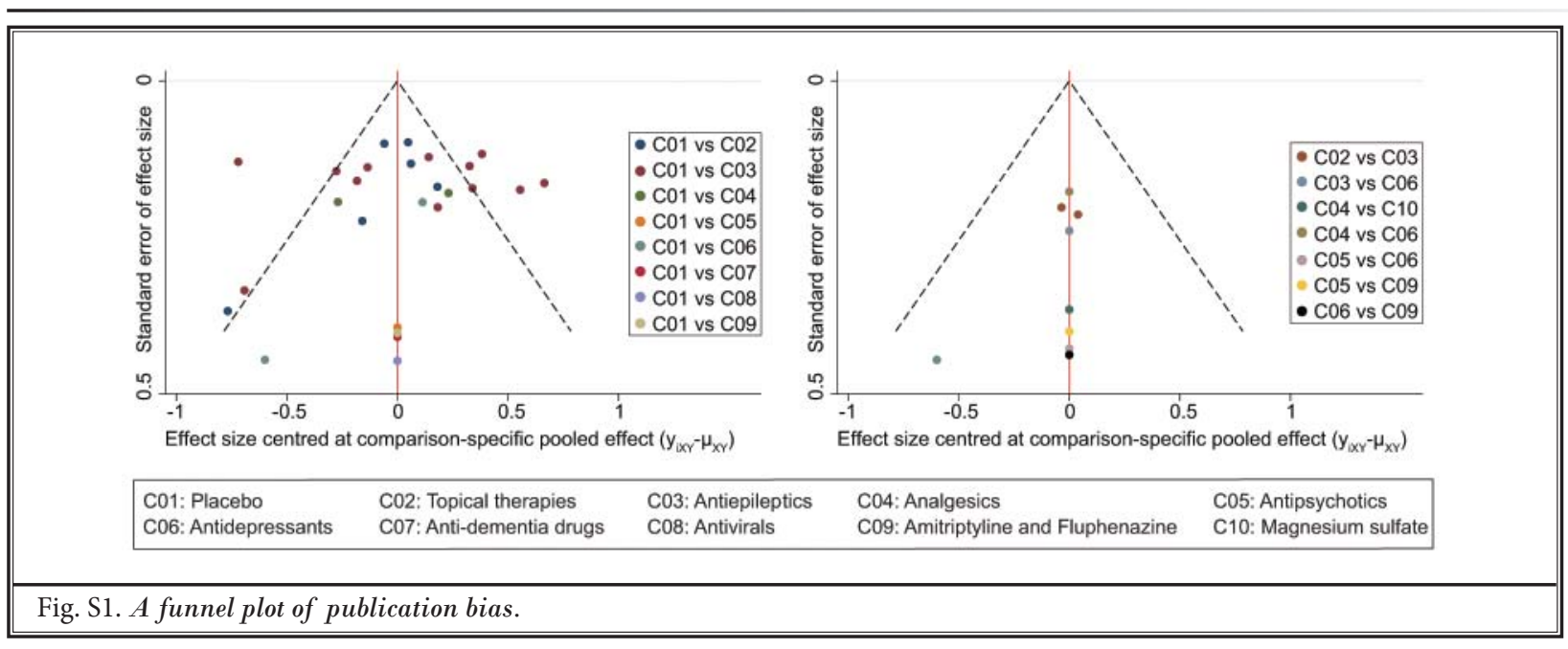


Pain Relief in PHN Therapies

Table S1. Node-splitting results of the network meta-analysis.

\begin{tabular}{|l|c|c|c|c|c|c|c||}
\hline \multirow{2}{*}{\multicolumn{1}{|c|}{ Class 1 vs Class 2 }} & \multicolumn{2}{c|}{ Direct } & \multicolumn{2}{c|}{ Indirect } & \multicolumn{3}{c|}{ Difference } \\
\cline { 2 - 9 } & Coef. & Std. Err. & Coef. & Std. Err. & Coef. & Std. Err. & \multicolumn{1}{c|}{ P } \\
\hline Placebo vs. Topical therapies & -0.29 & 0.19 & -0.85 & 0.37 & 0.55 & 0.41 & 0.18 \\
\hline Placebo vs. Antiepileptics & -0.71 & 0.13 & -0.22 & 0.33 & -0.49 & 0.36 & 0.17 \\
\hline Placebo vs. Analgesics & -0.63 & 0.34 & -1.82 & 1.16 & 1.20 & 1.21 & 0.32 \\
\hline Placebo vs. Antipsychotics & -0.28 & 0.60 & 0.80 & 1.28 & -1.07 & 1.42 & 0.45 \\
\hline Placebo vs. Antidepressants & -0.69 & 0.39 & -0.64 & 0.49 & -0.06 & 0.63 & 0.93 \\
\hline Placebo vs. A\&F & -0.31 & 0.60 & 0.77 & 1.28 & -1.07 & 1.42 & 0.45 \\
\hline Topical therapies vs. Antiepileptics & 0.15 & 0.34 & -0.41 & 0.23 & 0.55 & 0.41 & 0.18 \\
\hline Antiepileptics vs. Antidepressants & -0.17 & 0.51 & 0.06 & 0.39 & -0.23 & 0.65 & 0.73 \\
\hline Analgesics vs. Antidepressants & 0.41 & 0.48 & -0.54 & 0.62 & 0.94 & 0.78 & 0.23 \\
\hline Analgesics vs. Magnesium sulfate & -0.12 & 0.58 & 1.45 & 63.25 & -1.58 & 63.25 & 0.98 \\
\hline Antipsychotics vs. Antidepressants & -0.80 & 0.61 & 0.27 & 1.26 & -1.07 & 1.42 & 0.45 \\
\hline Antidepressants vs. A\&F & 0.77 & 0.62 & -0.30 & 1.26 & 1.07 & 1.42 & 0.45 \\
\hline
\end{tabular}

Abbreviation: A\&F, amitriptyline and fluphenazine. A $P$ value less than 0.05 indicated a significant difference.

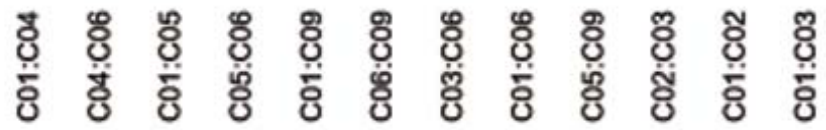

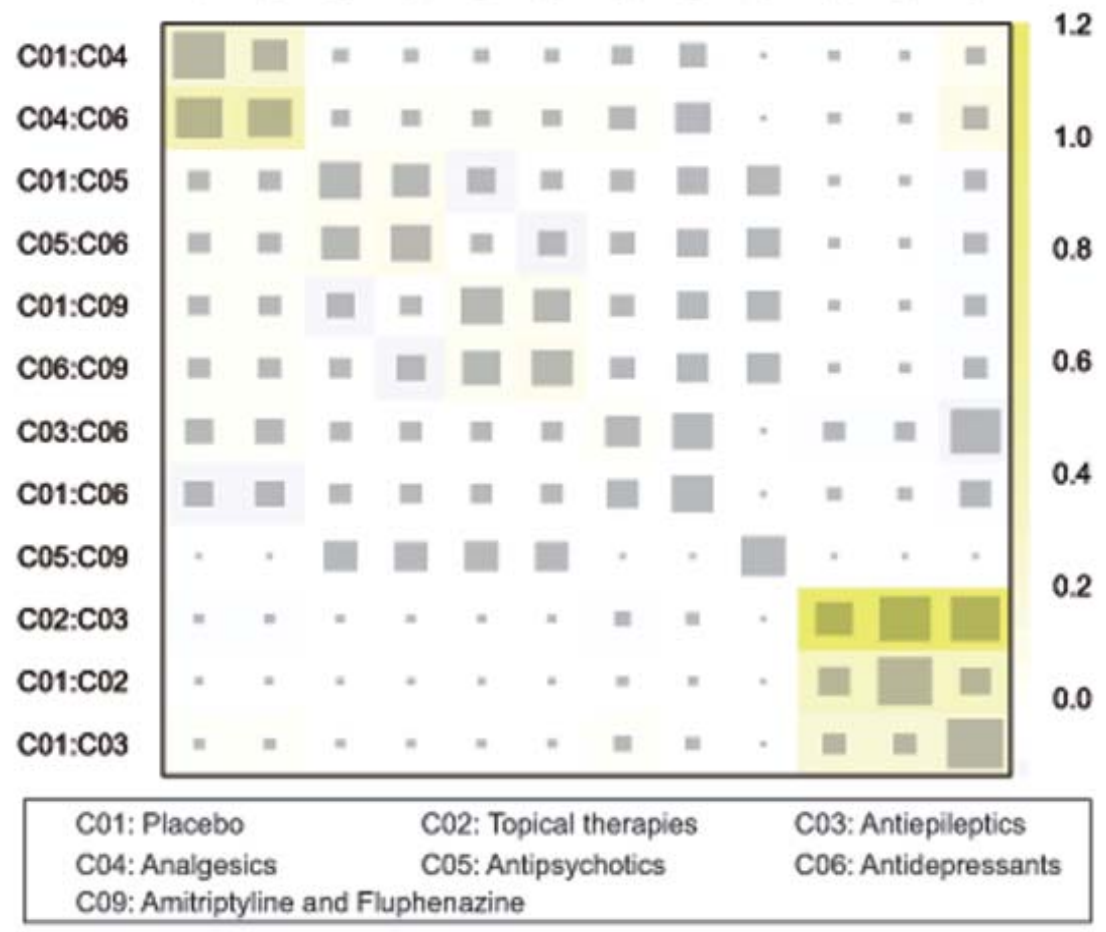

Fig. S2. A heat plot of the NMA. The area of a gray square displays the contribution of the direct estimate of one design in the column to a network estimate in a row. The colors on the diagonal represent the inconsistency contribution of the corresponding design. The colors on the off-diagonal are associated with the change in inconsistency between direct and indirect evidence in a network estimate in the row after relaxing the consistency assumption for the effect of one design in the column. 
Pain Physician: January/February 2018; 21:19-32

Table S2. SUCRA results and mean rank.

\begin{tabular}{|l|c|c||}
\hline \multicolumn{1}{|c|}{ Treatment } & Mean Rank & SUCRA $^{\mathbf{a}}$ \\
\hline Analgesics & 2.49 & 0.751 \\
\hline Magnesium sulfate & 2.64 & 0.736 \\
\hline Antidepressants & 3.60 & 0.640 \\
\hline Antiepileptics & 4.10 & 0.590 \\
\hline Topical therapies & 5.40 & 0.460 \\
\hline Anti-dementia drugs & 5.84 & 0.416 \\
\hline A\&F & 7.01 & 0.299 \\
\hline Antipsychotics & 7.51 & 0.249 \\
\hline Placebo & 7.72 & 0.228 \\
\hline Antivirals & 8.70 & 0.130 \\
\hline \hline
\end{tabular}

Abbreviations: SUCRA, surface under the cumulative ranking; A\&F, amitriptyline and fluphenazine. ${ }^{a}$ The treatments are sorted in descending order of SUCRA.

\section{References}

1. Sampathkumar P, Drage LA, Martin DP. Herpes zoster (shingles) and postherpetic neuralgia. Mayo Clin Proc 2009; 84:274-280.

2. Johnson RW, Wasner G, Saddier P, Baron R. Postherpetic neuralgia: Epidemiology, pathophysiology and management. Expert Rev Neurother 2007; 7:1581-1595.

3. Zin CS, Nissen LM, Smith MT, O'Callaghan JP, Moore BJ. An update on the pharmacological management of post-herpetic neuralgia and painful diabetic neuropathy. CNS Drugs 2008; 22:417-442.

4. Watson CP, Deck JH, Morshead C, Van der Kooy D, Evans RJ. Post-herpetic neuralgia: Further post-mortem studies of cases with and without pain. Pain 1991; 44:105-117.

5. Dworkin RH, Gnann JW Jr., Oaklander AL, Raja SN, Schmader KE, Whitley RJ. Diagnosis and assessment of pain associated with herpes zoster and postherpetic neuralgia. J Pain 2008; 9:S37-S44.

6. McCracken LM, Iverson GL. Disrupted sleep patterns and daily functioning in patients with chronic pain. Pain Res Manag 2002; 7:75-79.

7. London: National Institute for Health and Clinical Excellence (UK). Neuropathic pain: The pharmacological management of neuropathic pain in adults in non-specialist settings. National Institute for Health and Care Excellence: Clinical Guidelines, London 2013; www.nice. org.uk/guidance/CG173.

8. Davies PS, Galer BS. Review of lido- caine patch $5 \%$ studies in the treatment of postherpetic neuralgia. Drugs 2004; 64:937-947.

9. Backonja M, Wallace MS, Blonsky ER Cutler BJ, Malan P Jr., Rauck R, Tobias J; NGX-4010 C116 Study Group. NGX4010 , a high-concentration capsaicin patch, for the treatment of postherpetic neuralgia: A randomised, double-blind study. Lancet Neurol 2008; 7:1106-1112.

10. Oxman MN, Levin MJ, Johnson GR, Schmader KE, Straus SE, Gelb LD, Arbeit RD, Simberkoff MS, Gershon AA, Davis LE, Weinberg A, Boardman KD, Williams $\mathrm{HM}$, Zhang JH, Peduzzi PN, Beisel CE, Morrison VA, Guatelli JC, Brooks PA, Kauffman CA, Pachucki CT, Neuzil KM, Betts RF, Wright PF, Griffin MR, Brunell P, Soto NE, Marques AR, Keay SK, Goodman RP, Cotton DJ, Gnann JW Jr., Loutit J, Holodniy M, Keitel WA, Crawford GE, Yeh SS, Lobo Z, Toney JF, Greenberg RN, Keller PM, Harbecke R, Hayward AR, Irwin MR, Kyriakides TC, Chan CY, Chan IS, Wang WW, Annunziato PW, Silber JL; Shingles Prevention Study Group. $A$ vaccine to prevent herpes zoster and postherpetic neuralgia in older adults. $\mathrm{N}$ Engl J Med 2005; 352:2271-2284.

11. Graff-Radford SB, Shaw LR, Naliboff BN. Amitriptyline and fluphenazine in the treatment of postherpetic neuralgia. Clin J Pain 2000; 16:188-192.

12. Truini A, Cruccu G, Garcia-Larrea L. Painful sensory neuropathy. N Engl ] Med 2003; 349:306-307; author reply 306-307.

13. Liu Q, Chen H, Xi L, Hong Z, He L, Fu $\mathrm{Y}$, Fang H, Shang N, Yan P, Fan D. A ran- domized, double-blind, placebo-controlled trial to evaluate the efficacy and safety of pregabalin for postherpetic neuralgia in a population of Chinese patients. Pain Pract 2017; 17:62-69.

14. Smith T, DiBernardo A, Shi Y, Todd MJ, Brashear HR, Ford LM. Efficacy and safety of carisbamate in patients with diabetic neuropathy or postherpetic neuralgia: Results from 3 randomized, double-blind placebo-controlled trials. Pain Pract 2014; 14:332-342.

15. Surman OS, Flynn T, Schooley RT, Baer L, Parker S, Hirsch MS, Davis LG. A double-blind, placebo-controlled study of oral acyclovir in postherpetic neuralgia. Psychosomatics 1990; 31:287-292.

16. Kim YH, Lee PB, Oh TK. Is magnesium sulfate effective for pain in chronic postherpetic neuralgia patients comparing with ketamine infusion therapy? J Clin Anesth 2015; 27:296-300.

17. Wolff RF, Bala MM, Westwood M, Kessels AG, Kleijnen J. 5\% lidocaine-medicated plaster vs other relevant interventions and placebo for post-herpetic neuralgia (PHN): A systematic review. Acta Neurol Scand 2011; 123:295-309.

18. Dworkin RH, O'Connor AB, Backonja M, Farrar JT, Finnerup NB, Jensen TS, Kalso EA, Loeser JD, Miaskowski C, Nurmikko TJ, Portenoy RK, Rice AS, Stacey $B R$, Treede RD, Turk DC, Wallace MS. Pharmacologic management of neuropathic pain: Evidence-based recommendations. Pain 2007; 132:237-251.

19. Zakrzewska JM. Insufficient evidence to recommend topical lidocaine as first- 
line treatment for postherpetic neuralgia. Evid Based Dent 2007; 8:85-86.

20. Backonja MM, Canafax DM, Cundy KC. Efficacy of gabapentin enacarbil vs placebo in patients with postherpetic neuralgia and a pharmacokinetic comparison with oral gabapentin. Pain Med 2011; 12:1098-1108.

21. Backonja MM, Malan TP, Vanhove GF Tobias JK. NGX-4010, a high-concentration capsaicin patch, for the treatment of postherpetic neuralgia: A randomized, double-blind, controlled study with an open-label extension. Pain Med 2010; 11:600-608.

22. Baron R, Mayoral V, Leijon G, Binder A, Steigerwald I, Serpell M. $5 \%$ lidocaine medicated plaster versus pregabalin in post-herpetic neuralgia and diabetic polyneuropathy: An open-label, non-inferiority two-stage RCT study. Curr Med Res Opin 2009; 25:1663-1676.

23. Boureau F, Legallicier P, Kabir-Ahmadi M. Tramadol in post-herpetic neuralgia: A randomized, double-blind, placebocontrolled trial. Pain 2003; 104:323-331.

24. Chandra K, Shafiq N, Pandhi P, Gupta S, Malhotra S. Gabapentin versus nortriptyline in post-herpetic neuralgia patients: A randomized, double-blind clinical trial--the GONIP trial. Int ] Clin Pharmacol Ther 2006; 44:358-363.

25. Dworkin RH, Corbin AE, Young JP Jr., Sharma U, LaMoreaux L, Bockbrader H, Garofalo EA, Poole RM. Pregabalin for the treatment of postherpetic neuralgia: A randomized, placebo-controlled trial. Neurology 2003; 60:1274-1283.

26. Eisenberg E, Kleiser A, Dortort A, Haim T, Yarnitsky D. The NMDA (N-methyl-Daspartate) receptor antagonist memantine in the treatment of postherpetic neuralgia: A double-blind, placebo-controlled study. Eur J Pain 1998; 2:21-327.

27. Galer BS, Jensen MP, Ma T, Davies PS, Rowbotham MC. The lidocaine patch $5 \%$ effectively treats all neuropathic pain qualities: Results of a randomized, double-blind, vehicle-controlled, 3-week efficacy study with use of the neuropathic pain scale. Clin J Pain 2002; 18:297-301.

28. Irving $G$, Jensen $M$, Cramer $M, W u$ J, Chiang YK, Tark M, Wallace M. Efficacy and tolerability of gastric-retentive gabapentin for the treatment of postherpetic neuralgia: Results of a double-blind, randomized, placebo-controlled clinical trial. Clin J Pain 2009; 25:185-192.

29. Irving GA, Backonja MM, Dunteman E, Blonsky ER, Vanhove GF, Lu SP, To- bias J;NGX-4010 C117 Study Group. A multicenter, randomized, double-blind, controlled study of NGX-4010, a highconcentration capsaicin patch, for the treatment of postherpetic neuralgia. Pain Med 2011; 12:99-109.

30. Jensen MP, Chiang YK, Wu J. Assessment of pain quality in a clinical trial of gabapentin extended release for postherpetic neuralgia. Clin J Pain 2009; 25:286-292.

31. Kochar DK, Garg P, Bumb RA, Kochar SK, Mehta RD, Beniwal R, Rawat N. Divalproex sodium in the management of post-herpetic neuralgia: A randomized double-blind placebo-controlled study. QJM 2005; 98:29-34.

32. Raja SN, Haythornthwaite JA, Pappagallo M, Clark MR, Travison TG, Sabeen S, Royall RM, Max MB. Opioids versus antidepressants in postherpetic neuralgia: A randomized, placebo-controlled trial. Neurology 2002; 59:1015-1021.

33. Rehm S, Binder A, Baron R. Post-herpetic neuralgia: $5 \%$ lidocaine medicated plaster, pregabalin, or a combination of both? A randomized, open, clinical effectiveness study. Curr Med Res Opin 2010; 26:1607-1619.

34. Rice AS, Maton S. Gabapentin in postherpetic neuralgia: A randomised, double blind, placebo controlled study. Pain 2001; 94:215-224.

35. Rowbotham M, Harden N, Stacey B, Bernstein P, Magnus-Miller L. Gabapentin for the treatment of postherpetic neuralgia: A randomized controlled trial. JAMA 1998; 280:1837-1842.

36. Sabatowski R, Gálvez R, Cherry DA, Jacquot $F$, Vincent $E$, Maisonobe $P$, Versavel M; 1008-045 Study Group. Pregabalin reduces pain and improves sleep and mood disturbances in patients with post-herpetic neuralgia: Results of a randomised, placebo-controlled clinical trial. Pain 2004; 109:26-35.

37. van Seventer R, Feister HA, Young JP Jr., Stoker M, Versavel M, Rigaudy L. Efficacy and tolerability of twice-daily pregabalin for treating pain and related sleep interference in postherpetic neuralgia: A 13-week, randomized trial. Curr Med Res Opin 2006; 22:375-384

38. Wallace MS, Irving G, Cowles VE. Gabapentin extended-release tablets for the treatment of patients with postherpetic neuralgia: A randomized, double-blind, placebo-controlled, multicentre study. Clin Drug Investig 2010; 30:765-776.

39. Webster LR, Malan TP, Tuchman MM,
Mollen MD, Tobias JK, Vanhove GF. A multicenter, randomized, double-blind, controlled dose finding study of NGX4010, a high-concentration capsaicin patch, for the treatment of postherpetic neuralgia. J Pain 2010; 11:972-982.

40. Webster LR, Tark M, Rauck R, Tobias JK, Vanhove GF. Effect of duration of postherpetic neuralgia on efficacy analyses in a multicenter, randomized, controlled study of NGX-4010, an 8\% capsaicin patch evaluated for the treatment of postherpetic neuralgia. BMC Neurol 2010; 10:92.

41. Yu-Kang T. Node-splitting generalized linear mixed models for evaluation of inconsistency in network meta-analysis. Value Health 2016; 19:957-963.

42. Krahn U, Binder H, König J. Visualizing inconsistency in network meta-analysis by independent path decomposition. BMC Med Res Methodol 2014; 14:131.

43. Fashner J, Bell AL. Herpes zoster and postherpetic neuralgia: Prevention and management. Am Fam Physician 2011; 83:1432-1437.

44. Mauskop A, Altura BT, Cracco RQ, Altura BM. Intravenous magnesium sulphate relieves migraine attacks in patients with low serum ionized magnesium levels: A pilot study. Clin Sci (Lond) 1995 89:633-636.

45. Stannard CF, Porter GE. Ketamine hydrochloride in the treatment of phantom limb pain. Pain 1993; 54:227-230.

46. Gottrup H, Bach FW, Juhl G, Jensen TS. Differential effect of ketamine and lidocaine on spontaneous and mechanical evoked pain in patients with nerve injury pain. Anesthesiology 2006; 104:527-536.

47. Eide PK, Jørum E, Stubhaug A, Bremnes J, Breivik H. Relief of post-herpetic neuralgia with the $\mathrm{N}$-methyl-D-aspartic acid receptor antagonist ketamine: A double-blind, cross-over comparison with morphine and placebo. Pain 1994; 58:347-354.

48. Fields $\mathrm{HL}$, Rowbotham M, Baron $\mathrm{R}$. Postherpetic neuralgia: Irritable nociceptors and deafferentation. Neurobiol Dis 1998; 5:209-227.

49. Kvarnström A, Karlsten R, Quiding $\mathrm{H}$ Emanuelsson BM, Gordh T. The effectiveness of intravenous ketamine and lidocaine on peripheral neuropathic pain. Acta Anaesthesiol Scand 2003; 47:868-877.

50. Felsby S, Nielsen J, Arendt-Nielsen L, Jensen TS. NMDA receptor blockade in chronic neuropathic pain: A comparison of ketamine and magnesium chloride. 
Pain 1996; 64:283-291.

51. Attal N, Cruccu G, Baron R, Haanpää $M$, Hansson P, Jensen TS, Nurmikko T; European Federation of Neruological Soicieties. EFNS guidelines on the pharmacological treatment of neuropathic pain: 2010 revision. Eur ] Neurol 2010; 17:1113-e1188.

52. Chen N, Li Q, Yang J, Zhou M, Zhou D, He L. Antiviral treatment for preventing postherpetic neuralgia. Cochrane Database Syst Rev 2014; CDoo6866.
53. Li Q, Chen N, Yang J, Zhou M, Zhou D, Zhang Q, He L. Antiviral treatment for preventing postherpetic neuralgia. Cochrane Database Syst Rev 2009; 15:CDoo6866. 